\title{
Effects of P-MAPA Immunotherapy Associated with Gemcitabine on Chemically-Induced Pancreatic Cancer in Animal Model: New Therapeutic Perspectives
}

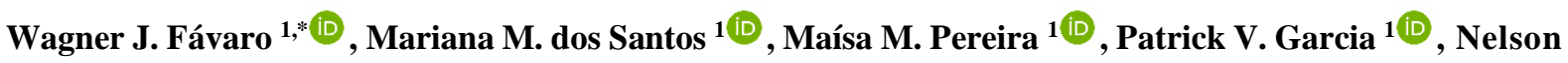 \\ Durán 1,2,*(D)
}

1 Laboratory of Urogenital Carcinogenesis and Immunotherapy, Department of Structural and Functional Biology, University of Campinas (UNICAMP), Campinas, SP, Brazil; wjfavaro@gmail.com (W.J.F.); martinis.mariana@gmail.com (M.M.S.); maisa_massafera@hotmail.com (M.M.P.); viannapatrick@yahoo.com.br (P.V.G.);

2 Nanomedicine Research Unit (Nanomed), Federal University of ABC (UFABC), Santo André, SP, Brazil; nduran@unicamp.br (N.D.);

* Correspondence: nduran@unicamp.br; (N.D); wjfavaro@gmail.com (W.J.F);

Scopus Author ID 23072832700

Received: 13.10.2021; Revised: 10.11.2021; Accepted: 14.11.2021; Published: 25.11.2021

\begin{abstract}
Pancreatic cancer is one of the most aggressive tumors since it accounts for approximately $5 \%$ of cancer-related deaths worldwide. Immunotherapy based on compounds capable of acting as tolllike receptor (TLRs) agonists may be a valuable strategy to treat cancer, either alone or in association with prevailing therapies. Thus, P-MAPA (Protein aggregate magnesium-ammonium phospholinoleatepalmitoleate anhydride) has emerged as a likely candidate to treat some cancer types, such as pancreatic cancer (PC). The current study reports the effects of an emerging alternative therapy against PC, which lies in associating P-MAPA immunotherapy with gemcitabine-based chemotherapy to treat $P C$ in murine models. Besides, the study reports the potential mechanisms of action of this new therapeutic association involving the TLR4 signaling pathway. PC chemically induced in animal model based on 7,12-dimethylbenz(a)anthracene carcinogen administered by thermosensitive copolymer effectively induced pancreatic tumors in $100 \%$ of the investigated rats. P-MAPA-based immunotherapy application alone has shown histopathological repair in $40 \%$ of rats, whereas those only treated with gemcitabine have shown $100 \%$ of malignant tumors. P-MAPA/Gemcitabine-associated treatment was highly effective in reducing neoplastic lesion progression and enabling histopathological improvement in $80 \%$ of the investigated rats. P-MAPA and P-MAPA/Gemcitabine treatments led to increased TLR4 protein contents, which led to increased interferon signaling pathways and positive antitumor effectiveness due to suppressed abnormal cell proliferation. Thus, it is a possible conclusion that the P-MAPA immunotherapy/gemcitabine association had a positive effect on murine models with PC and that it may be a valuable alternative to treat this tumor type.
\end{abstract}

Keywords: Immunotherapy; Toll-like Receptors; Pancreatic Cancer; P-MAPA; Gemcitabine.

Abbreviations: BSA: bovine serum albumin; DMBA: 7,12-Dimethylbenz(a)anthracene; HRP: horseradish peroxidase; IFN- $\gamma$ : interferon-gamma; IL: interleukin; IL-6: interleukin 6; IRF-3: Interferon

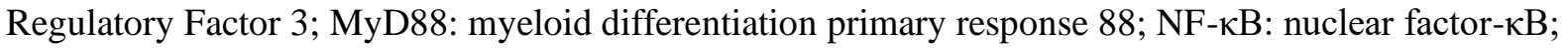
PC: pancreatic cancer; PanIN: Pancreatic Intraepithelial Neoplasia; P-MAPA: Protein Aggregate Magnesium-Ammonium Phospholinoleate-Palmitoleate Anhydride; TLR: Toll-Like Receptor; TNF- $\alpha$ : tumor necrosis factor $\alpha$; TRAIL: TNF-related-apoptosis-inducing ligand; TRIF: TIR-domaincontaining adapter-inducing interferon- $\beta$.

(C) 2021 by the authors. This article is an open-access article distributed under the terms and conditions of the Creative Commons Attribution (CC BY) license (https://creativecommons.org/licenses/by/4.0/). 


\section{Introduction}

Pancreatic cancer (PC) is one of the most significant and aggressive tumors [1] since it accounts for approximately 5\% of cancer-related deaths worldwide [2]. Smoking habit is the most important factor leading to pancreatic cancer, although the disease can also be associated with other factors such as individuals' exposure to certain metal types [3], caloric intake and/or obesity [4, 5], aging [6], as well as with some chronic conditions such as diabetes, chronic pancreatitis and cirrhosis [7, 8], and with genetic factors that account for $10 \%$ of PC cases [6]. Most PC (95\%) cases comprise adenocarcinomas, which are highly aggressive tumors [3]. Early PC diagnosis is hard because of its aggressiveness and lack of early disease-specific symptoms [6, 9].

Understanding pancreatic carcinogenesis mechanisms and enabling the progress of prevention methods are paramount priority challenges; thus, several factors capable of causing PC evolution have been investigated in murine models [10-13]. Chemical carcinogens can induce approximately $70 \%$ of human pancreatic adenocarcinomas [14]. Several chemical agents are capable of inducing cancer in animals, but 7,12-dimethylbenz(a)anthracene (DMBA) is the one mostly used in rodents [15-18]. The use of mice models with malignant tumors in recent years has significantly contributed to better understanding human tumors and evaluating new early disease detection, prevention, and treatment strategies [19-23]. Nowadays, surgical resection is merely an option for PC treatment. PC patients subjected to full surgical resection present a 5-year survival rate of approximately $25 \%$. Chemotherapy and radiation are palliative treatments adopted to help increase patients' likelihood of survival or improve their quality of life [3].

Some safe and sufficiently tested adjuvant therapies are currently used against cancer. Among them, one finds 5-Fluorouracil (5-FU) [24-26] and gemcitabine [27]. Studies have compared the effectiveness of 5-FU and gemcitabine in improving PC patients' response--totreatment rates. Gemcitabine has shown an increased survival rate and better benefits to patients compared to those recorded for patients treated with 5-FU [28]. Therefore, clinical trials have focused on exploring the pharmacokinetic modulation of gemcitabine, in combination with other cytotoxic or biological agents [2], and found some significant associations $[29,30]$.

Besides enhancing antigen emergence and immune checkpoint expression, gemcitabine has significantly increased the synthesis of CCL/CXCL chemokines and TGF $\beta$-associated signals. These factors account for gemcitabine's resistance to cancer-associated fibroblasts in vitro and enhanced TGF $\beta 1$ biosynthesis. Gemcitabine and anti-PD-1 were only efficient in rats subjected to previous TGF $\beta$ signaling oblation. Such a condition has led to significant CD8+ $\mathrm{T}$ - cell response, diminished tumor load, and enabled longer survival time. Data have suggested that gemcitabine is capable of priming pancreatic tumors for immune checkpoint inhibition by enhancing antigen presentation only following disruption of the immunosuppressive cytokine barrier [31].

The use of erlotinib (immunomodulatory drug capable of inhibiting the epidermal growth factor receptor tyrosine kinase) [32] associated with gemcitabine has been successfully used to treat severe PC. Thus, it was considered an alternative to help enhance the survival of patients with early-stage resectable PC who did not present microscopic evidence of cancer cells at the primary tumor site. An open-label (CVONKO-005 trial), the multicenter trial has assigned patients to one of two treatments, namely: gemcitabine + erlotinib or gemcitabine 
(Gem), alone. The first aim of the trial was to enhance disease-free survival (DFS) based on the erlotinib/gemcitabine combination. There was no significant difference in median DFS or median overall survival between patients treated with gemcitabine/erlotinib and Gem. There was a trend towards long-term survival in patients treated with gemcitabine/erlotinib combinations compared to those treated with Gem. It is important to emphasize that this trial was the first to investigate the association between chemotherapy and specific therapy (targeted) as adjuvant PC treatment [33]. However, immunomodulation/gemcitabine association can be an excellent strategy adopted to treat pancreatic cancer.

Compounds capable of acting on Toll-like receptors (TLRs) are valuable approaches to cancer treatment, either used separately or in association with current therapies [34,35]. TLRs play a key role in innate immunity since they stimulate individuals' immune systems to act on tumor cells and eliminate the system inhibiting the immune machinery [36-38]. MyD88- and TRIF-dependent are two TLR signaling pathways [36-38]. MyD88-dependent pathway triggers NF-kB and MAPK, and it releases inflammatory cytokines such as TNF- $\alpha$ (tumor necrosis factor $\alpha$ ) and IL-6 [36,38]. IRF-3 (TRIF-dependent pathway) activates interferon regulatory factor 3 in order to generate interferons [36-38]. TLR4 operates all four adapter molecules (MyD88, TRAM, TRIF, and TIRAP) in cascade signals [36-38].

Immunotherapy is an important strategy adopted to treat cancer patients [35]. Accordingly, P-MAPA has emerged as a potential candidate for such treatment (Patent Number: PI0305373-3, 2003) [20, 39]. Experiments conducted with HEK293 cells in vitro have shown that the P-MAPA treatment applied to these cells was capable of activating NF$\kappa \mathrm{B}$ and, consequently, activating TLRs 2 and 4 [20]. Garcia et al. [40] have shown that bladdercancer animal models subjected to intravesical immunotherapy with P-MAPA presented TLRs 2 and 4-mediated innate immune system activation, which led to increased interferon signaling pathways (TRIF, IFN- $\alpha$, IRF-3, and IFN- $\gamma$ ) and p53 protein contents. The aforementioned authors have concluded that P-MAPA acted as TLRs 2 and 4 agonists, activated p53 protein, and improved the immunological status of bladder cancer animal models [40].

The aim of the current study was to describe the outcomes of a potential alternative treatment for PC cases based on the application of P-MAPA immunotherapy in association with gemcitabine chemotherapy to the PC murine model. Besides, the study supports the likely mechanisms of this therapeutic association in the TLR4 signaling pathway.

\section{Materials and Methods}

\subsection{Experimental procedures.}

The current pre-clinical experiment was conducted with 25 male rats (Fischer 344 strain), who were 12 weeks old and weighed approximately 200 grams; animals were provided by the University of Campinas (CEMIB/ UNICAMP)[40]. The experimental protocol has strictly followed the ethical principles for animal research (CEUA/IB/ UNICAMP-protocol number: 3631-1). PC induction was performed in 20 male rats, who were intraperitoneally (i.p.) anesthetized with $60 \mathrm{mg} / \mathrm{Kg}$ of $10 \%$ ketamine (Vibra ${ }^{\circledR}$ Roseira, São Paulo, Brazil) and 5 $\mathrm{mg} / \mathrm{Kg}$ of $2 \%$ xylazine (Vibra ${ }^{\circledR}$ Roseira, São Paulo, Brazil), and subjected to the transverse incision in the upper abdominal wall (epigastric region, Figure 1a) in order to expose their abdominal organs. Next, delicate dissection was performed to separate the stomach from the transverse colon to identify the pancreas and its regions (Figure 1b). Subsequently, the head of the pancreas was isolated and subjected to intraparenchymal application of a single dose of 200 
$\mu \mathrm{g}$ dimethylbenzanthracene (DMBA - Sigma, St. Louis, MO, USA) dissolved in $200 \mu \mathrm{l}$ acetone, with the aid of $13.0 \mathrm{~mm}$ X $0.45 \mathrm{~mm}$ needle. The carcinogen (DMBA) was administered through a thermosensitive copolymer (Pluronic 127, Sigma, St. Louis, MO, USA). The carcinogen/thermosensitive copolymer mixture has formed a gel (Patent Registration Number: BR1020140231188), which was introduced right into the head of the pancreas (Figure 1c). Abdominal wall synthesis was performed with 3-0 monofilament nylon thread (Shalon Medical, Montes Claros, Goiás, Brazil) after pancreatic cancer induction (Figure 1d).

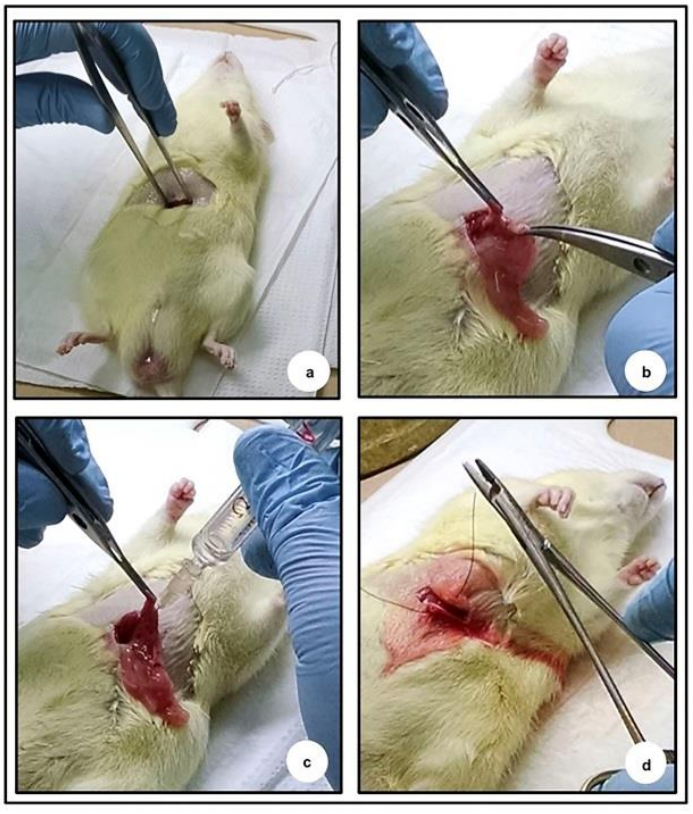

Figure 1. Surgical procedure for pancreatic cancer induction. (a) The transverse incision in the upper abdominal wall (epigastric region) exposes abdominal organs. (b) Delicate dissection to separate the stomach and transverse colon, followed by identifying the pancreas and its regions. (c) Intraparenchymal application of thermosensitive copolymer containing DMBA carcinogen in the head of the pancreas. (d) Synthesis of the abdominal wall.

The control group comprised 5 animals who did not receive thermosensitive copolymer added with a carcinogen. After 120 days of PC induction, all animals were subjected to ultrasound examination to investigate the tumor incidence; they were divided into 5 groups (5 animals per group). The control group (Group 1) received $0.3 \mathrm{~mL}$ of $0.9 \%$ physiological saline solution intraperitoneally delivered for 6 consecutive weeks; the DMBA group (Cancer, Group 2) received the same treatment as Group 1; the DMBA + P-MAPA group (Group 3) received $5 \mathrm{mg} / \mathrm{Kg}$ of P-MAPA (Farmabrasilis, São Paulo, Brazil) intraperitoneally delivered three times a week, for 6 consecutive weeks; the DMBA + Gemcitabine group (Group 4) received 10 $\mathrm{mg} / \mathrm{Kg}$ of Gemcitabine intraperitoneally delivered once a week, for 6 consecutive weeks [41]; and the DMBA+ P-MAPA + Gemcitabine group (Group 5) received $5 \mathrm{mg} / \mathrm{Kg}$ of P-MAPA three times a week, for 3 consecutive weeks - after that, this group received $10 \mathrm{mg} / \mathrm{Kg}$ of Gemcitabine intraperitoneally delivered once a week, for 3 consecutive weeks (6-week treatment, in total).

Rats were euthanized after 6 weeks of treatment to collect pancreatic samples, which were processed for histopathological, immunological, and Western Blotting analysis.

\subsection{Histopathological analysis.}


Pancreatic fragments were collected from 5 rats in each group and fixed in Bouin's solution for 12 hours [40]. Next, they were washed in $70 \%$ ethanol and subjected to dehydration based on ascending alcohol series. Subsequently, these fragments were diaphanized in xylene for 2 hours and embedded in the plastic polymer (Paraplast Plus, ST. Louis, MO, USA); 5- $\mu$ mthick specimens were cut in rotary microtome Slee CUT5062 RM 2165 (Slee Mainz, Mainz, Germany), stained with hematoxylin-eosin and photographed in DM2500 (Leica, Munich, Germany) photomicroscope. Pancreatic lesions were diagnosed by a veterinary pathologist based on morphological criteria described by Hruban et al. [42].

\subsection{Proliferative and apoptotic index determination.}

Samples similar to the ones subjected to histopathological analysis were used to determine proliferative and apoptotic indices. Ki-67 immunodetection was used to determine the proliferative index [40]. Specimens were cut into 5- $\mu \mathrm{m}$-thick sections, and the antigen was extracted by boiling the sections in citrate buffer $(10 \mathrm{mM}, \mathrm{pH} 6.0)$ three times for $5 \mathrm{~min}$ (microwave oven). Next, the sections were incubated in $0.3 \% \mathrm{H}_{2} \mathrm{O}_{2}$ to block endogenous peroxidase; nonspecific binding was blocked by incubating the aforementioned sections in blocking solution at room temperature. Primary rat monoclonal anti-Ki-67 antibody (NCLKi67-MM1, Novocastra; Newcastle, United Kingdom; 1:50) was diluted in 1\% BSA and applied to the sections overnight at $4^{\circ} \mathrm{C}$. Bound antibody was detected with the aid of the Advance $^{\mathrm{TM}}$ HRP kit (Dako Cytomation Inc., USA). The sections were lightly counterstained with Harris' hematoxylin and photographed in DM2500 (Leica, Munich, Germany) photomicroscope. Ten fields were randomly photographed under $\times 40$ objective lens and measured per animal, which resulted in 50 fields per group; the total number of Ki-67-stained positive cells was expressed as a rate of total cells.

DNA fragmentation (TUNEL) was evaluated through Terminal Deoxynucleotidyl Transferase (TdT) by using FragELTM DNA Kit (Calbiochem, La Jolla, CA, USA) [40]. Apoptotic nuclei were identified based on diaminobenzidine chromogen mixture (FragELTM DNA Kit). Up to ten microscopic fields were randomly selected and analyzed per sample (50 fields per group) in Leica DM2500 (Leica, Munich, Germany) photomicroscope equipped with $\times 40$ objective lens. Sections were lightly counterstained with Harris' hematoxylin. The apoptotic index was determined by dividing the number of apoptotic nuclei by the total number of nuclei observed in the microscope field [40].

\subsection{Western blotting analysis of toll-like receptor 4 (TLR4), IRF-3, and IFN- $\gamma$.}

Pancreatic samples $(n=5)$ of each group were weighed $(\sim 200 \mathrm{mg})$ and homogenized in $50 \mu \mathrm{L} / \mathrm{mg}$ of RIPA lysis buffer (EMD Millipore Corporation, Billerica, MA, USA) [40]. Samples presenting $10 \mu \mathrm{g}$ of protein were separated through SDS-PAGE using $12 \%$ polyacrylamide gels under reducing conditions. After the electrophoresis was over, proteins were transferred to Hybond-ECL nitrocellulose membranes (Amersham, Pharmacia Biotech, Arlington Heights, IL., USA), which were blocked with RAPIDBLOCK ${ }^{\mathrm{TM}}$ SOLUTION (Amresco, Fountain Parkway Solon, OH, EUA) diluted in Ultra-pure water (1:10) for 5 minutes and incubated overnight $\left(\right.$ at $4^{\circ} \mathrm{C}$ ) with primary rat monoclonal anti-TLR4 (sc-293072, Santa Cruz Biotechnology, Inc., Santa Cruz, CA, USA), rat monoclonal anti-IRF3 (sc-376455, Santa Cruz Biotechnology, Inc., Santa Cruz, CA, USA) and rat monoclonal anti-IFN- $\gamma$ (507802; Biolegend, USA) antibodies diluted in $1 \%$ BSA. Next, membranes were incubated with rat 
secondary HRP-conjugated antibodies (diluted 1:1,000 in $1 \%$ BSA; Santa Cruz Biotechnology, Inc., Santa Cruz, CA, USA) for 2 hours. Peroxidase activity was detected through incubation with diaminobenzidine chromogen (Sigma Chemical Co., St Louis, USA). Western blots were run in duplicate; pancreas samples were collected from 5 rats per group for each repetition. Semi-quantitative densitometry (IOD - Integrated Optical Density) analysis of bands was conducted in NIH ImageJ $1.47 \mathrm{v}$ software (National Institute of Health, USA. Available at: http://rsb.info.nih.gov/ij/), and it was followed by statistical analysis. $\beta$-actin (monoclonal rat, A5441, Sigma Chemical Co., St Louis, USA) was applied as an endogenous positive control to standardize band-staining intensity readings. Data were expressed as mean \pm standard deviation of each band intensity/ $\beta$-actin band intensity ratio [40].

\subsection{Statistical analysis.}

All parameters, i.e., Western Blotting, proliferative and apoptotic indices, and proliferation/apoptotic ratio (P/A) - were statistically compared among groups based on analysis of variance, which was followed by Turkey's test at $1 \%$ and $5 \%$ significance levels [40]. Data were expressed as mean \pm standard deviation. Histopathological analyses were based on proportion tests. The difference between the two proportions was subjected to Fisher's exact test. The type-I error of $1 \%$ was considered statistically significant for all analyses.

\section{Results and Discussion}

\subsection{Thermosensitive hydrogel added with DMBA carcinogen was effective in inducing} pancreatic cancer in Fischer 344 rats.

The present results have shown that any animal in the Control group presented malignant lesions (Table 1). The macroscopic analysis applied to surgical specimens deriving from the Control group has evidenced normal-sized pancreas and enabled differentiating pancreatic regions such as head, body, and tail (Figure 2a).

Table 1. Percentage of histopathological changes in the pancreas of animals from different experimental groups.

\begin{tabular}{c|c|c|c|c|c} 
Histopatology & Group 1 $(\mathbf{n}=\mathbf{5})$ & Group 2 $(\mathbf{n = 5})$ & Group 3 $(\mathbf{n}=\mathbf{5})$ & Group 4 (n= 5) & Group 5 (n=5) \\
\hline Normal & $5(100 \%)^{*}$ & - & - & - & $3(60 \%)$ \\
\hline Ductal Hyperplasia & - & - & $2(40 \%)^{*}$ & - & $1(20 \%)$ \\
\hline PanIN 1 & - & - & $2(40 \%)^{*}$ & $1(20 \%)$ & $1(20 \%)$ \\
\hline PanIN 2 & - & - & $1(20 \%)^{*}$ & $1(20 \%)^{*}$ & - \\
\hline PanIN 3 & - & $2(40 \%)^{*}$ & - & $2(40 \%)^{*}$ & - \\
\hline Invasive Carcinoma & - & $3(60 \%)^{*}$ & - & $1(20 \%)$ & -
\end{tabular}

Groups: Control (Group 1), DMBA (Group 2), DMBA + P-MAPA (Group 3), DMBA + Gemcitabine (Group

4), and DMBA + P-MAPA + Gemcitabine (Group 5).

Pancreatic Intraepithelial Neoplasia (PanIN): 1, 2, 3

*Statistical significance (Ratio test, $P<0.0001$ )

The microscopic analysis applied to this very same group has evidenced pancreas is composed of rounded serous acini presenting basophilic cells, which represent the exocrine region of the gland. In addition, it was possible seeing an islet of Langerhans featuring the endocrine region (Figure 3a). Moreover, interlobular ducts presented simple cuboidal epithelium surrounded by stroma rich in collagen fibers and blood vessels (Figure 3b). 


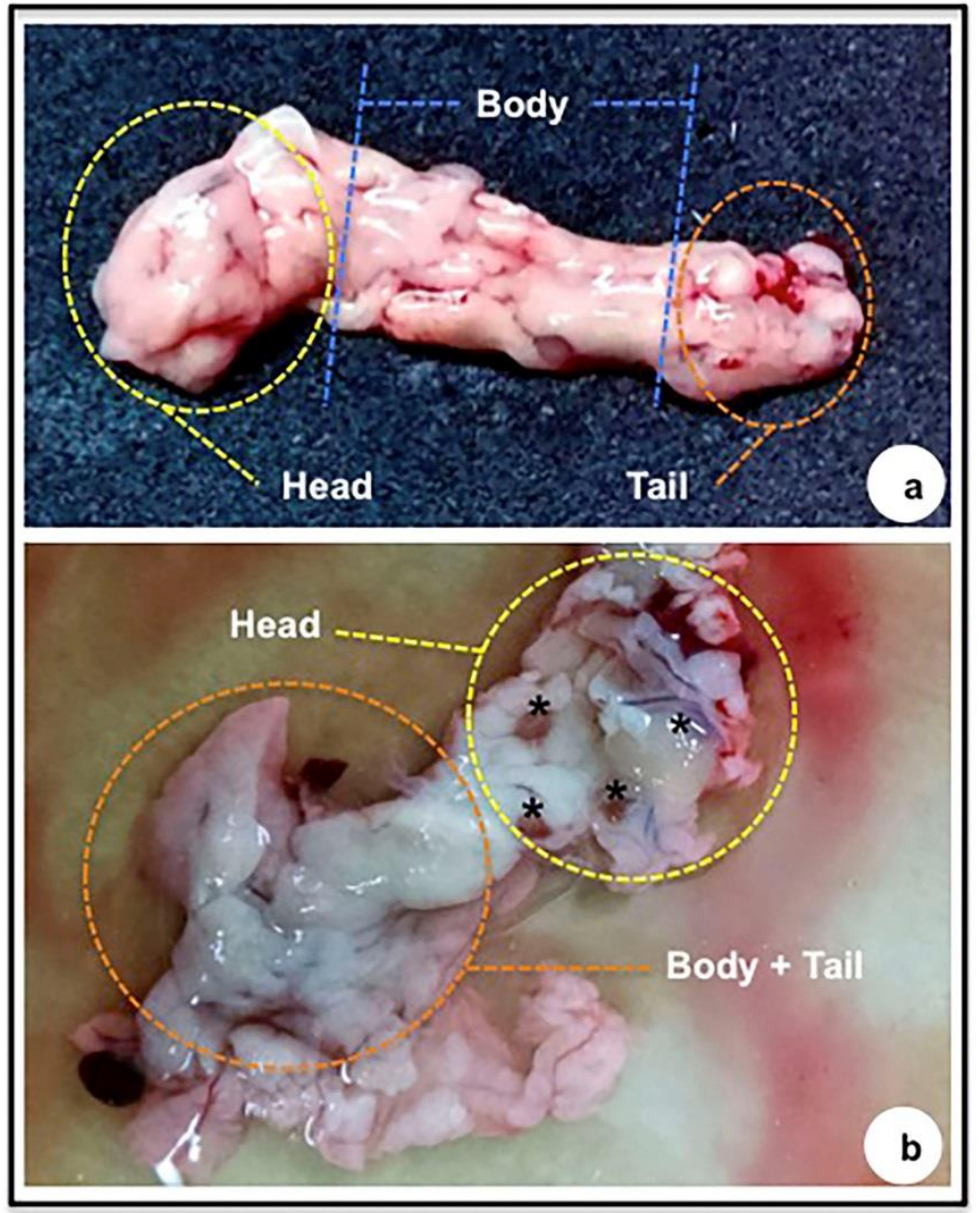

Figure 2. Gross anatomy of the pancreas from (a) Control and (b)DMBA Group. (a) Pancreas with normal size, being possible to distinguish the regions of the head, body, and tail. (b) Bulky pancreas, being very difficult to distinguish body and tail regions; neoplastic nodular lesions (asterisks) in the head of the pancreas.

Macroscopic analysis of surgical specimens has shown that DMBA-induced animals presented bulky pancreas, a fact that made it very difficult to distinguish pancreatic body and tail regions (Figure 2b). In addition, the head of the pancreas has shown neoplastic nodular lesions (Figure 2b).

Highly-frequent histopathological changes observed in the pancreas of the DMBA group comprised Grade-3 Pancreatic Intraepithelial Neoplasia (PanIN-3) (Figures 3c, 3d) and invasive carcinoma (Figures 3e, 3f) in $40 \%$ and $60 \%$ of rats, respectively (Table 1). PanIN-3 was featured by papillary or micropapillary lesions with high-grade dysplasia; cell nuclei were bulky, pleomorphic, and showed cellular polarity loss (Figures 3c, 3d, 3h). Some PanIN-3 cases presented detached cell groups heading towards the lumen of the ducts. Invasive carcinoma (Figures 3e, 3f, 3i, 3j) was featured by neoplastic cells presenting intense cellular atypia, arranged in nests or cords that infiltrated the desmoplastic stroma. Neoplastic cell nuclei were bulky, pleomorphic, and presented evident nucleoli.

Overall, a significant number of risk factors were associated with PC progression, such as exposure to chemical carcinogens. Smoking habit is one of the worst risk factors for this cancer type [11,43] since tobacco smoke has approximately 4,700 chemicals, and some of them, such as DMBA, are highly genotoxic [44]). Studies have shown that DMBA is a strong mutagenic agent that leads to skin, mouth, breast, and pancreatic tumors [16, 45, 46]. Dissin et al. [45] and Bockman et al. [17] have found well-differentiated pancreatic adenocarcinoma in $63.3 \%$ and $70.0 \%$ of animals chemically induced with DMBA, respectively. Similarly, Rivera 
et al. [16] have shown that hyperplasia (39.0\%), low-grade dysplasia (8.0\%), high-grade dysplasia (10.0\%), and pancreatic ductal adenocarcinoma (39.0\%) were the histopathological changes mostly observed in the pancreas of rats induced with DMBA.

Thus, the DMBA carcinogen herein administered through thermosensitive copolymer (Patent registration number: BR1020140231188) - in order to chemically induce PC development in animal models - was effective in inducing pancreatic tumors in $100 \%$ of animals. The PC murine model presented specific advantages for experimental data on total carcinogenesis since carcinogen administered through thermosensitive copolymer can be delivered in quantifiable pulse doses. In addition, this autochthonous PC model has other advantages such as low cost (non-dependent on high-cost technologies), reproducibility, and immunocompetent host, which is crucial in studies about compounds capable of acting on the immune system.

\subsection{P-MAPA immunotherapy associated with Gemcitabine chemotherapy reverses} histopathological changes induced by DMBA.

Gemcitabine treatment has reduced the aggressiveness of malignant lesions induced by DMBA. However, rats only treated with gemcitabine have shown 100\% malignant damages. Histopathological changes observed in this group lied on PanIN-1, PanIN-2 (Figure 3g), PanIN-3 (Figure 3h), and Invasive Carcinoma (Figures 3i, 3j), which were found in 20\%, 20\%, $40 \%$, and $20 \%$ of rats, respectively (Table 1 ).

PanIN-1 (Figures 4b, 4f) was featured by flat lesions with low-grade dysplasia, composed of columnar epithelial cells presenting uniform nuclei perpendicular to the basement membrane of ducts. PanIN-2 (Figures 3g, 4c) was morphologically more complex than PanIN1 since it showed moderate dysplasia grade, nuclei (enlarged in size and number) presenting cell polarity loss, hyperchromasia, and pseudostratification.

On the other hand, P-MAPA therapy application alone has effectively reduced the progression of pancreatic neoplastic lesions. Ductal hyperplasia (Figure 4a), PanIN-1 (Figure 4b), and PanIN-2 (Figure 4c) was the histopathological changes mostly observed in $40 \%, 40 \%$, and $20 \%$ of rats, respectively (Table 1 ).

Rats treated with P-MAPA in combination with gemcitabine presented better histopathological repair from the rats exhibiting cancer than the ones in the DMBA+P-MAPA and DMBA + Gemcitabine groups since the P-MAPA + Gemcitabine treatment has reduced the progression of pancreatic neoplastic lesions in $80 \%$ of rats (Figures $4 \mathrm{~d}, 4 \mathrm{e}, 4 \mathrm{f}$; Table 1). Normal ductal morphology (Figure 4d) was observed in $60 \%$ of rats. Ductal hyperplasia (Figure 4e) and PanIN-1 (Figure 4f) were the histopathological changes mostly found in this group - $20 \%$ and $20 \%$ of rats, respectively (Table 1 ).

Chang et al. [46] have shown that Gemcitabine chemotherapy in combination with TLR2 agonist (rlipo-E7m/CpG) has led to a significant decrease in pancreatic tumor size. Similarly, Ghansah et al. [21] have shown a significant decrease in tumor growth in rats subjected to treatment based on the combination between gemcitabine and dendritic cell vaccine immunotherapy.

Treatment based on the combination between triacyl lipid A (OM-174) and cyclophosphamide chemotherapy was more effective in reducing tumor growth than treatments based on OM-174 or cyclophosphamide application separate [43]. 


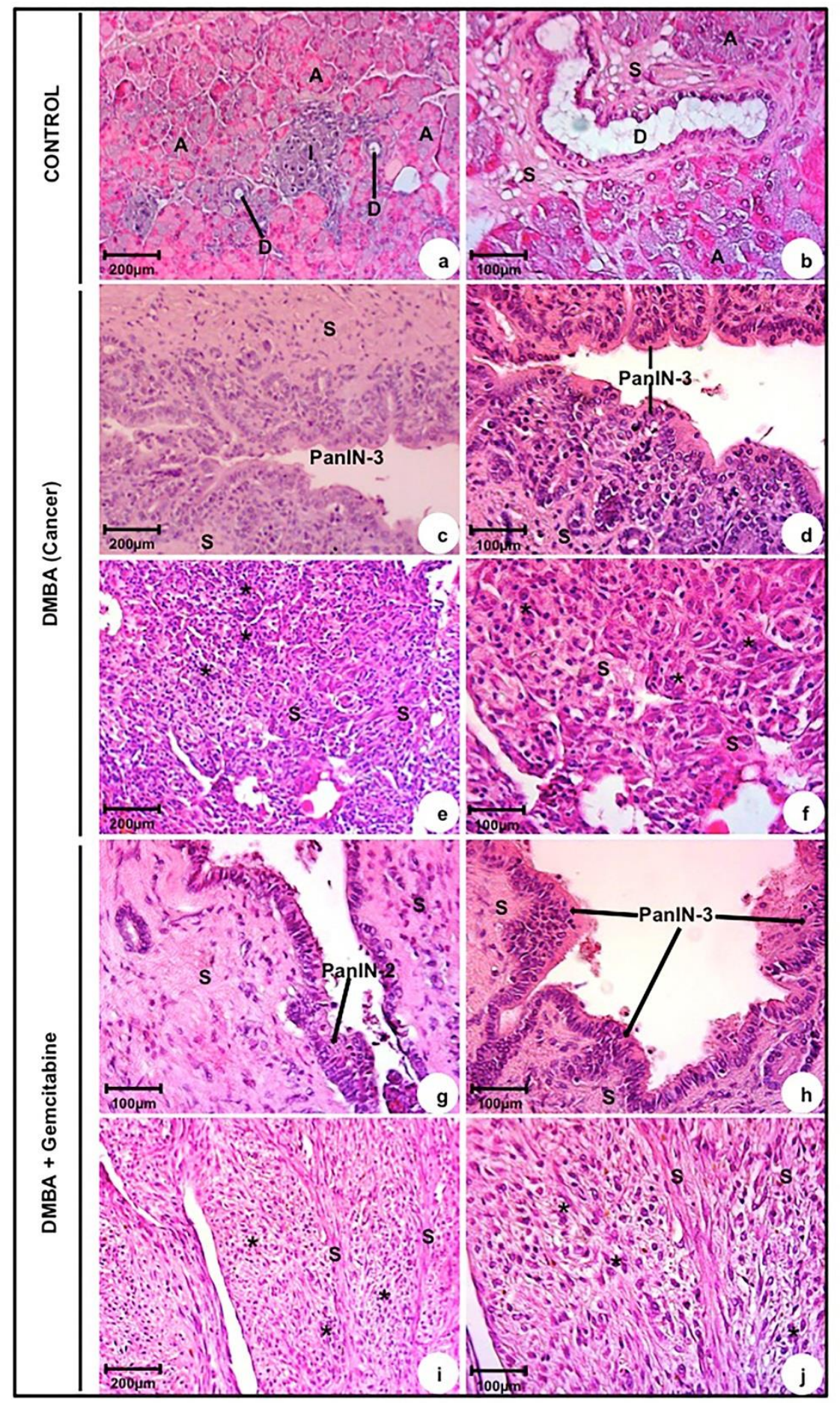

Figure 3. Photomicrographs of the pancreas from (a, b) Control, (c, d, e, f) DMBA, and (g, h, i, j) DMBA + Gemcitabine groups. (a), (b) Pancreas composed by rounded serous acini (A) containing basophilic cells, representing the exocrine region of the gland; islet of Langerhans (I), characterizing the endocrine region; interlobular ducts (D) composed by simple cuboidal epithelium, surrounded by stroma (S) rich in collagen fibers and blood vessels. (c), (d), (h) PanIN-3 characterized by papillary or micropapillary lesions with high-grade dysplasia; the nuclei were bulky, pleomorphic, and showed loss of cellular polarity; stroma (S). (e), (f), (i), (j) Invasive carcinoma characterized by neoplastic cells with intense cellular atypia, arranged in nests or cords (asterisks) infiltrating the desmoplastic stroma (S); neoplasic cells with bulky nuclei, pleomorphic and with evident nucleoli. (g) PanIN-2 is characterized by a moderate grade degree of dysplasia; nuclei enlarged in size and number, with loss of cell polarity, hyperchromasia, and pseudostratification. 


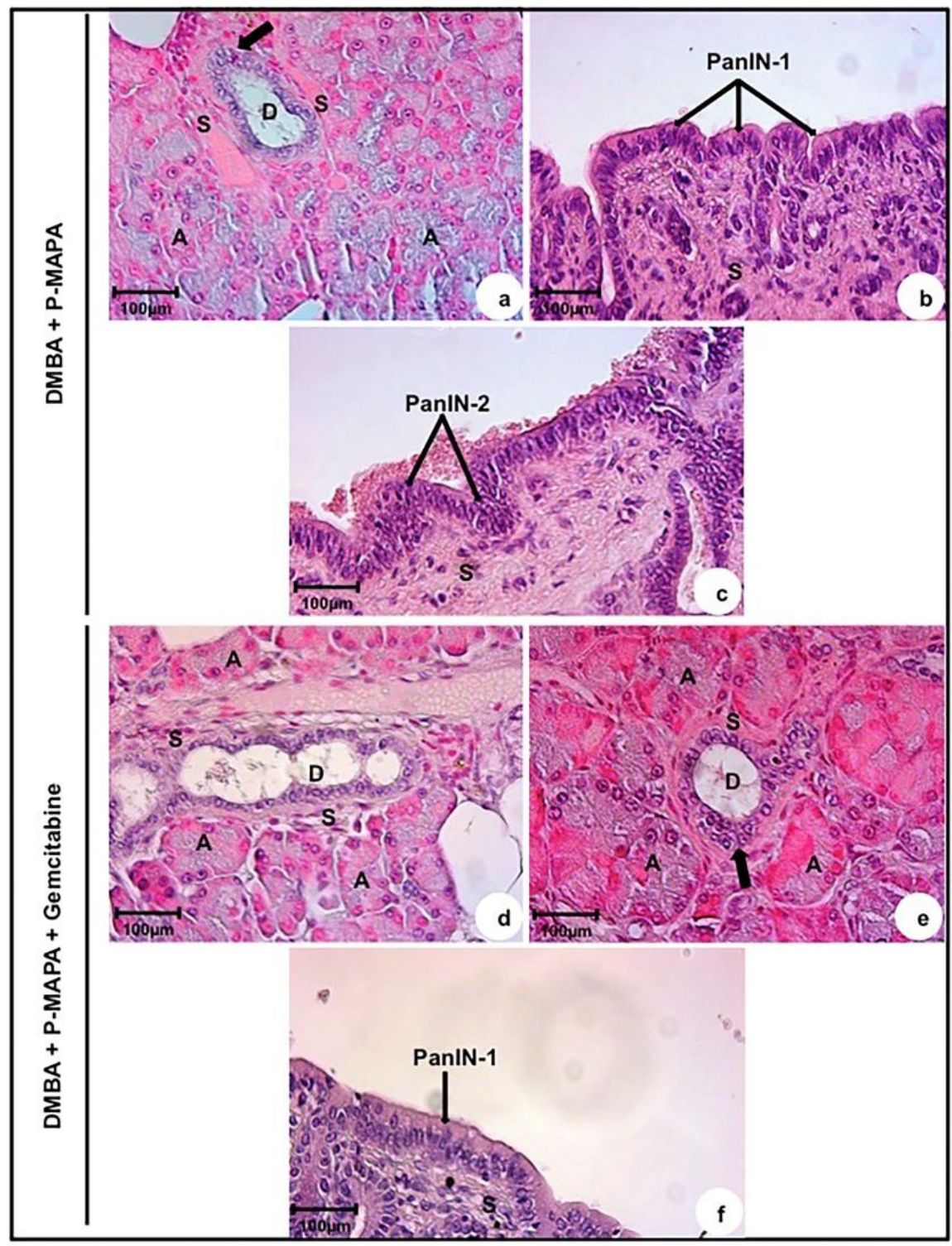

Figure 4. Photomicrographs of the pancreas from (a, b, c) DMBA + P-MAPA and (d, e, f) DMBA + P-MAPA + Gemcitabine groups. (a), (e) Ductal hyperplasia characterized by proliferation of cuboidal ductal cells (arrow); Interlobular ducts $(\mathbf{D})$ rounded serous acini (A) and stroma (S). (b), (f) PanIN-1 is characterized by flat lesions with low-grade dysplasia, composed of columnar epithelial cells with uniform nuclei oriented perpendicular to the basement membrane of ducts. (c) PanIN-2 characterized by moderate grade degree of dysplasia; nuclei enlarged in size and number, with loss of cell polarity, hyperchromasia, and pseudostratification; (d) Pancreas composed by rounded serous acini (A) containing basophilic cells; interlobular ducts (D) composed by simple cuboidal epithelium, surrounded by stroma $(\mathbf{S})$ rich in collagen fibers and blood vessels.

Clinical studies have shown positive results in PC patients subjected to immunotherapeutic associations [18, 47]. According to Dias et al. [22], the association of intravesical P-MAPA immunotherapy with cisplatin (systemic) has reduced the progression of urothelial neoplastic damages and enabled histopathological repair in $80 \%$ of rats with chemically-induced bladder cancer.

Although the association between chemotherapies is the alternative most often selected to elaborate treatment regimens focused on pancreatic tumors, the association between chemotherapy and immunotherapy can also be applied to treat patients who are not suitable for pancreatectomy, as well as to the ones presenting unfavorable conditions to operate locally advanced and metastatic PC [48]. Therefore, the combination between immunotherapy and chemotherapy is a potential evolution in PC treatment. 
Thus, the current study has shown that the association between P-MAPA immunotherapy and Gemcitabine in PC murine model was effective, reasonably tolerated, and did not show apparent antagonism signs between drugs.

3.3. P-MAPA immunotherapy in association with Gemcitabine chemotherapy activates the interferon signaling pathway, decreases disease proliferation, and increases apoptosis.

The most elevated TLR4 protein levels were found in the DMBA + P-MAPA, DMBA + P-MAPA + gemcitabine, and Control groups in comparison to the other experimental groups (Figure 5a). In addition, DMBA and DMBA + Gemcitabine groups did not show significant differences in TLR4 protein levels (Figure 5a).

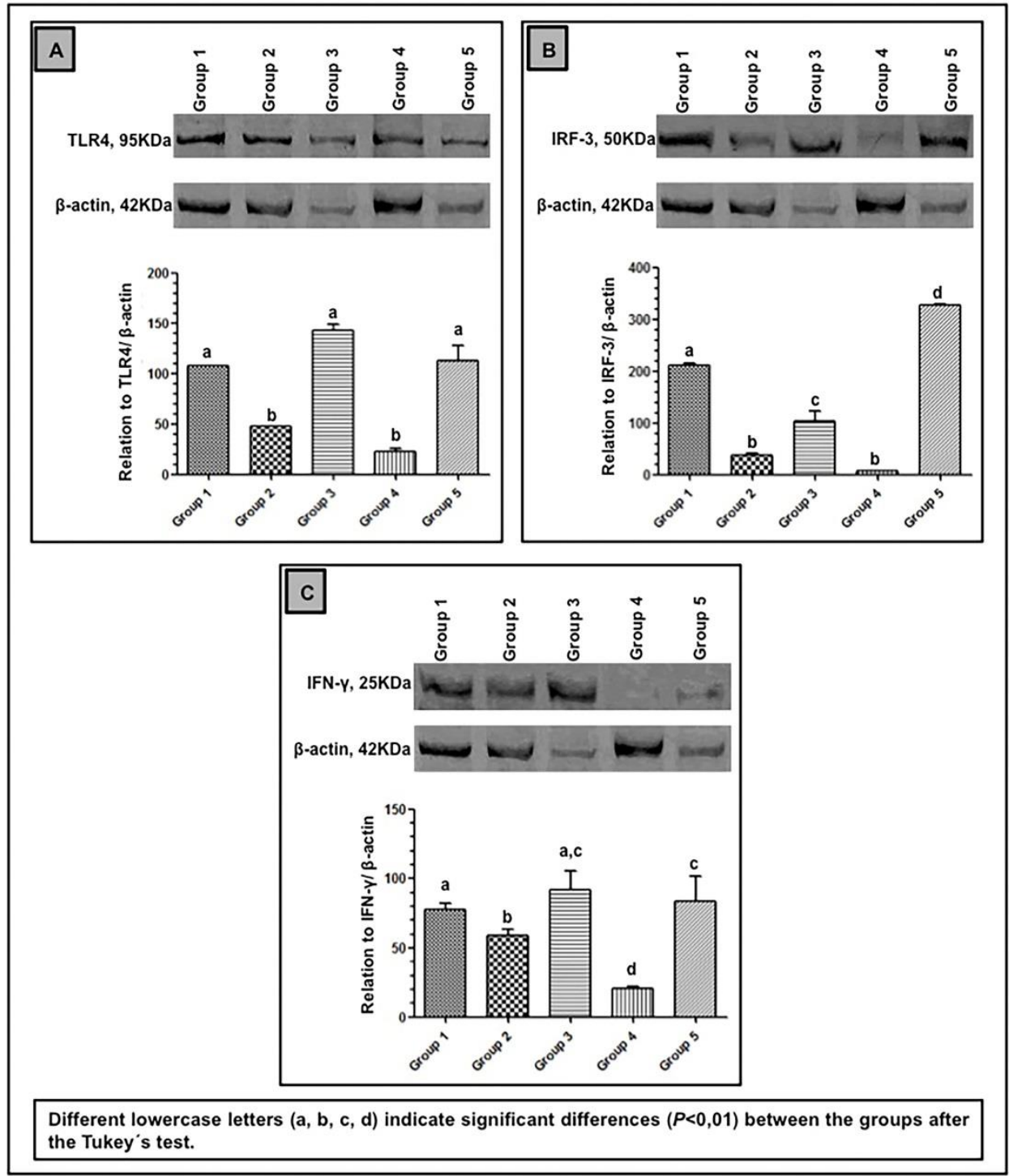

Figure 5. Western Blotting and semi-quantitative determination for (a) TLR4, (b) IRF-3, and (c) IFN- $\gamma$ protein levels. Specimens of the pancreas were pooled from five rats per group for each repetition (duplicate) and utilized for semi-quantitative densitometry (IOD - Integrated Optical Density) analysis of the TLR4, IRF-3, and IFN- $\gamma$ levels following normalization to the $\beta$-actin. Groups: Control (Group 1); DMBA (Group 2); DMBA + PMAPA (Group 3); DMBA + Gemcitabine (Group 4); DMBA + P-MAPA + Gemcitabine (Group 5). Data were depicted as the mean \pm standard deviation. Different lowercase letters $(\mathbf{a}, \mathbf{b}, \mathbf{c}, \mathbf{d})$ indicate significant differences $(\mathrm{p}<0.01)$ between the groups after Tukey's test.

IRF-3 protein levels were significantly higher in the DMBA + P-MAPA + gemcitabine group than in the other experimental groups (Figure 5b). In addition, these levels were 
significantly higher in the DMBA + P-MAPA group than in the Control, DMBA, and DMBA + Gemcitabine groups (Figure 5b).

The highest IFN- $\gamma$ protein levels were found in the DMBA + P-MAPA + Gemcitabine and DMBA + P-MAPA groups compared to the Control group (Figure 5c). In addition, these contents were significantly higher in the DMBA group than in the DMBA + Gemcitabine group (Figure 5c).

Moreover, the current study has shown that the proliferative effect has significantly increased in rats belonging to the DMBA and DMBA + gemcitabine groups compared to the other experimental groups (Figure 6a). DMBA + P-MAPA + Gemcitabine, DMBA + P-MAPA, and Control groups have shown significantly lower mean proliferative index values (Figure $6 a)$.

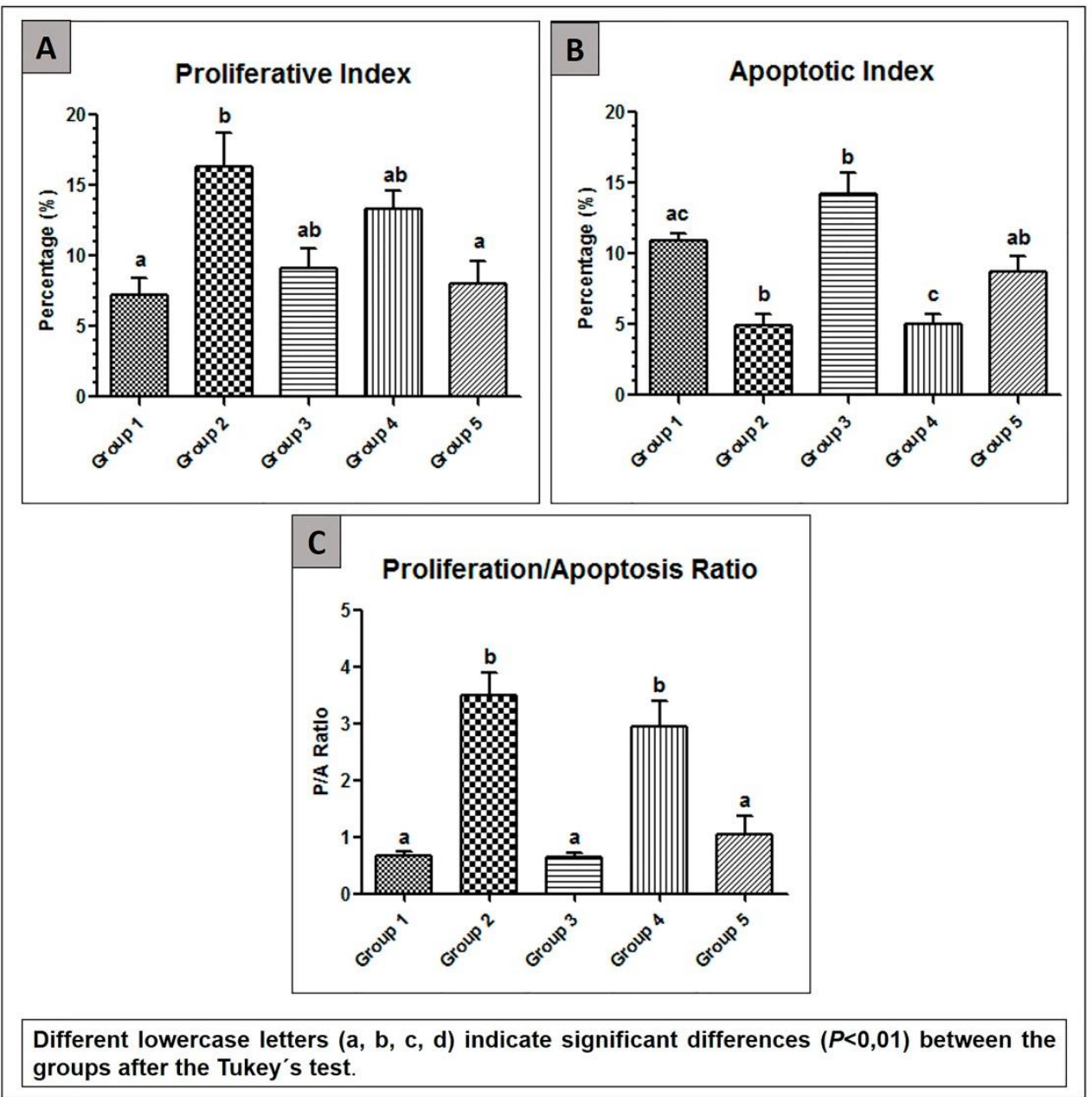

Figure 6. (a) Percentage of Proliferative Index; (b) Percentage of Apoptotic Index; (c) Proliferation/Apoptotic Ratio (P/A). Groups: Control (Group 1); DMBA (Group 2); DMBA + P-MAPA (Group 3); DMBA +

Gemcitabine (Group 4); DMBA + P-MAPA + Gemcitabine (Group 5). The data were depicted as mean \pm SD. Different lowercase letters $(\mathbf{a}, \mathbf{b}, \mathbf{c}, \mathbf{d})$ indicate significant differences $(\mathrm{p}<0.05)$ between the groups after Tukey's test.

The apoptotic index has shown different cell death kinetics in all treatments (Figure $6 b)$. This index was significantly higher in rats belonging to the DMBA + P-MAPA group than in rats belonging to the other groups. On the other hand, DMBA + P-MAPA + Gemcitabine and Control groups have shown notably higher mean apoptotic index values than the DMBA and DMBA + Gemcitabine groups (Figure 6b). 
Moreover, the proliferation/apoptotic ratio (P/A) was significantly lower in the DMBA + P-MAPA + Gemcitabine and DMBA + P-MAPA groups than in the other groups; these two groups have shown the prevalence of apoptotic process (Figure 6c).

TLRs agonists are an important research topic and innovation in cancer treatments, such as bladder cancer [20,40,49]. TLRs play a crucial role in activating innate and adaptive immune responses [49]. TLR4 activation in host macrophages has generated different inflammatory cytokines capable of affecting tumor growth. However, TLR4 signaling has produced cytokines (IFN) that have antitumor effects due to TRAIL (TNF-related-apoptosisinducing ligand) induction - TRAIL is an effective tumor cell death inducer [50]. Tumor suppressor activity performed by the immune system strongly depends on IFN- $\gamma$ activity, which, at least in part, regulates tumor-cell immunogenicity [51]. IFN- $\gamma$ stimulates several antiproliferative and tumoricidal mechanisms in macrophages and tumor cell lines; besides, it also has a strong impact on solid tumor growth and metastasis and likely plays an initial role in preventing metastasis development [52-54].

According to D'Agostini et al. [43], OM-174 (TLRs 2 / 4 antagonist) has decreased tumor progression and increased the survival rate of rats with B16 melanoma. Furthermore, a recent study has shown that OM-174 was unable to induce tumor regression in both IFN- $\gamma$ and TLR4 knockout animals with induced breast cancer [55]. Garcia et al. [40] have shown that PMAPA intravesical immunotherapy applied to bladder cancer animal models has activated TLRs 2 and 4-mediated innate immune system and led to increased interferon signaling pathway (TRIF, IRF-3, IFN- $\alpha$, and IFN- $\gamma$ ), which was fundamental for histopathological recovery from cancer stage.

Thus, it is possible saying that interferon signaling pathway activation caused by $\mathrm{P}$ MAPA treatment alone, or associated with gemcitabine, has increased apoptosis and decreased abnormal cell proliferation, which resulted in histopathological recovery.

\section{Conclusions}

Data analyzed in the conducted experiments indicate that interferon signaling pathway activation by P-MAPA immunotherapy application alone and/or associated with Gemcitabine chemotherapy may enable new immunotherapeutic approaches to PC.

\section{Funding}

This research did not receive any specific grant from funding agencies in the public, commercial, or not-for-profit sectors.

\section{Acknowledgments}

The authors thank CNPq-Brazil (grant nos. 490519/2011-3 and 475211/2013-8) and FAPESPBrazil (grant nos. 2011/05726-4, 2012/20706-2, 2012/13585-4, 2014/20465-0 and 2014/11866-1).

\section{Conflicts of Interest}

The authors declare that they have no known competing financial interests or personal relationships that could have influenced the work reported in this paper. 


\section{References}

1. Park, W.; Chawla, A.; O'Reilly, EM. Pancreatic cancer. A Review. JAMA. 2021, 326, 851-862, https://doi.org/10.1001/jama.2021.13027.

2. Sung, H.; Ferlay, J.; Siegel, R.L.; Laversanne, M.; Soerjomataram, I.; Jemal, A.; Bray, F. Global Cancer Statistics 2020: GLOBOCAN Estimates of Incidence and Mortality Worldwide for 36 Cancers in 185 Countries. CA Cancer J Clin 2021,71, 209-249, https://doi.org/10.3322/caac.21660.

3. Pamphlett, R.; Colebatch, A.J.; Doble, P.A.; Bishop, D.P. Mercury in Pancreatic Cells of People with and without Pancreatic Cancer. Int J Environ Res Public Health 2020, 17, 8990, https://doi.org/10.3390/ijerph17238990.

4. Genkinger, J.M.; Spiegelman, D.; Anderson, K.E.; Bernstein, L.; van den Brandt, P.A.; Calle, E.E.; English, D.R.; Folsom, A.R.; Freudenheim, J.L.; Fuchs, C.S.; Giles, G.G.; Giovannucci, E.; Horn-Ross, P.L.; Larsson, S.C.; Leitzmann, M.; Mannisto, S.; Marshall, J.R.; Miller, A.B.; Patel, A.V.; Rohan, T.E.; StolzenbergSolomon, R.Z.; Verhage, B.A.; Virtamo, J.; Willcox, B.J.; Wolk, A.; Ziegler, R.G.; Smith-Warner, S.A. A pooled analysis of 14 cohort studies of anthropometric factors and pancreatic cancer risk. Int J Cancer 2011, 129, 1708-1717, https://doi.org/10.1002/ijc.25794.

5. Eibl, G.; Rozengurt, E. Obesity and pancreatic cancer: Insight into mechanisms. Cancers 2021, 13, 5067, https://doi.org/10.3390/cancers13205067.

6. Xie, D.; Qian, B.; Yang, J.; Peng, X.; Li, Y.; Teng Hu, T.; Lu, S.; Chen, X.; Han, Y. Can Elderly Patients With Pancreatic Cancer Gain Survival Advantages Through More Radical Surgeries? A SEER-Based Analysis. Front Oncol 2020, 10, 598048, https://doi.org/10.3389/fonc.2020.598048.

7. Menini, S.; Iacobini, C.; Vitale, M.; Pesce, C.; Pugliese, G. Diabetes and pancreatic cancer-A dangerous liaison relying on carbonyl stress. Cancers 2021, 13, 313, https://doi.org/10.3390/cancers13020313.

8. de Rezende, A.Q.; Gestic, M.A.; Utrini, M.P.; Chaim, F.D,M.; de Saito, H.A. ; Chaim, E.A.; Callejas-Neto, F.; Cazzo, E. Is there a link between non-alcoholic fatty liver disease aspects and pancreatic cancer? Results of a case-matched study. Rev Col Bras Cir 2021, 48, e20202913, https://doi.org/10.1590/0100-6991e20202913

9. Kleeff, J.; Korc, M.; Apte, M.; La Vecchia, C.; Johnson, C.D.; Biankin, A.V.; Neale, R.E.; Tempero, M.; Tuveson, D.A., Hruban, R.H.; Neoptolemos, J.P. Pancreatic cancer. Nat Rev Dis Primers 2016, 2, 22, https://doi.org/10.1038/nrdp.2016.22.

10. Mallya, K.; Gautam, S.K.; Aithal, A.; Batra, S.K.; Jain, M. Modeling pancreatic cancer in mice for experimental therapeutics. BBA - Reviews on Cancer 2021, 1876, 188554, https://doi.org/10.1016/j.bbcan.2021.188554.

11. Ozcan-Sınır, G.; Inan, S.; Suna, S.; Tamer, C.E.; Akgul, M.B.; Bagdas, D.; Sonmez, G.; Evrensel, T.; Kaya, E.; Sarandol, E.; Dundar, H.Z.; Tarım, O.F.; Ercan, I.; Sıgıri, D.; Incedayi, B.; Copur, O.U. Effect of high fructose corn sirup on pancreatic ductal adenocarcinoma induced by dimethyl benzantracene (DMBA) in rats. Nutrition Cancer 2021, 73, 339-349, https://doi.org/10.1080/01635581.2020.1770811.

12. Okusaka, T.; Furuse, J. Recent advances in chemotherapy for pancreatic cancer: evidence from Japan and recommendations in guidelines. J Gastroenterol 2020, 55,369-382, https://doi.org10.1007/s00535-02001666-y.

13. Looi, C-K.; Chung, FF-L.; Leong, C-O.; Wong, S-F.; Rosli, R.; Mai, C-W. Therapeutic challenges and current immunomodulatory strategies in targeting the immunosuppressive pancreatic tumor microenvironment. J Exper Clin Cancer Res 2019, 38,162, https://doi.org/10.1186/s13046-019-1153-8.

14. Principe, D.R.; Underwood, P.W.; Korc, M.; Trevino, J.G.;. Munshi, H.G.; Rana, A.

The Current Treatment Paradigm for Pancreatic Ductal Adenocarcinoma and Barriers to Therapeutic Efficacy. Front Oncol 2021, 11, 688377, https://doi.org/10.3389/fonc.2021.688377.

15. Jimenez, R.E.; Z'Graggen, K.; Hartwig, W.; Graeme-Cook, F.; Warshaw, A.L.; Fernandez-del Castillo, C. Immunohistochemical characterization of pancreatic tumors induced by dimethylbenzanthracene in rats. $\mathrm{Am}$ J Pathol 1999, 154, 1223-1229, https://doi.org/10.1016/S0002440(10)65374-6.

16. Kogawa, T.; Sato, Y.; Shimoyama, R.; Kamakura, S.; Tashiro, Y.; Yoshiro Niitsu, Y. Chemoprevention of Pancreatic Cancer by Inhibition of Glutathione-S Transferase P1 . Research Square. Preprint 2021, https://doi.org/10.21203/rs.3.rs-408015/v1.

17. Bockman, D.E.; Guo, J.; Buchler, P.; Muller, M.W.; Bergmann, F.; Friess, H. Origin and development of the precursor lesions in experimental pancreatic cancer in rats. Lab Invest 2003, 83, 853-859, https://doi.org/10.1097/01.LAB.0000074918.31303.5A.

18. Kimura, Y.; Tsukada, J.; Tomoda, T.; Takahashi, H.; Imai, K.; Shimamura, K.; Sunamura, M.; Yonemitsu, Y.; Shimodaira, S.; Koido, S.; Homma, S.; Okamoto, M. Clinical and immunologic evaluation of dendritic cell-based immunotherapy in combination with gemcitabine and/or S-1 in patients with advanced pancreatic carcinoma. Pancreas 2012, 41, 195-205, https://doi.org/10.1097/MPA.0b013e31822398c6.

19. Kachi, K.; Kato, H.; Naiki-Ito, A.; Komura, M.; Nagano-Matsuo, A.; Naitoh, I.; Hayashi, K.; Kataoka, H.; Inaguma, S.; Takahashi, S. Anti-allergic drug suppressed pancreatic carcinogenesis via down-regulation of cellular proliferation. Int J Mol Sci 2021, 22, 7444, https://doi.org/10.3390/ijms22147444. 
20. Favaro, W.J.; Nunes, O.S.; Seiva, F.R.; Nunes, I.S.; Woolhiser, L.K.; Durán, N.; Lenaerts, A.J. Effects of PMAPA immunomodulator on toll-like receptors and p53: Potential therapeutic strategies for infectious diseases and cancer. Infect Agent Cancer 2012,7, 14, https://doi.org/10.1186/1750-9378-7-14.

21. Ghansah, T.; Vohra, N.; Kinney, K.; Weber, A.; Kodumudi, K.; Springett, G.; Sarnaik, A.A.; Pilon-Thomas, S. Dendritic cell immunotherapy combined with gemcitabine chemotherapy enhances survival in a murine model of pancreatic carcinoma. Cancer Immunol Immunother 2013, 62, 1083-1091, https://doi.org/10.1007/s00262-013-1407-9.

22. Dias, Q.C.; Nunes, I.D.; Garcia, P.V.; Favaro, W.J. Potential therapeutic strategies for non - muscle invasive bladder cancer based on association of intravesical immunotherapy with $\mathrm{p}$-MAPA and systemic administration of cisplatin and doxorubicin. Int Braz J Urol 2016, 42, 942-954, https://doi.org/10.1590/S1677-5538.IBJU.2015.0381.

23. Prasad, S.; Yadav, V.R.; Sung, B.; Gupta, S.C.; Tyagi, A.K.; Aggarwal, B.B. Ursolic acid inhibits the growth of human pancreatic cancer and enhances the antitumor potential of gemcitabine in an orthotopic mouse model through suppression of the inflammatory microenvironment. Oncotarget 2016, 7, 13182-13196, https://doi.org/10.18632/oncotarget.7537.

24. Liu, G.; Yang, L.; Chen, G.; Xu, F.; Yang, F.; Yu, H.; Li, L.; Dong, X.; Han, J.; Cao, C.; Qi, J.; Su, J.; Xu, X.; Li, X.; Li, B. A review on drug delivery system for tumor therapy. Front Pharmacol 2021, 12, 735446, https://doi.org/10.3389/fphar.2021.735446.

25. Yoo, C.; , Kim,K-P.; Kim, I.; Kang, M.J.; J. Kang, B.W.; Ryu, H.; Jeong, J.H.; Lee, J.S.; Kim, K.W.; Ryoo, B-Y. Liposomal irinotecan (nal-IRI) in combination with fluorouracil (5-FU) and leucovorin (LV) for patients with metastatic biliary tract cancer (BTC) after progression on gemcitabine plus cisplatin (GemCis): Multicenter comparative randomized phase 2b study (NIFTY). J Clinl Oncol 2021. 39, 4006-4006, https://doi.org/10.1200/JCO.2021.39.15_suppl.4006.

26. Ghafouri-Fard, S.; Abak, A.; Anamag, F.B.; Shoorei,m H.; Fattahi, F.; Javadinia, S.A.; Basiri, A.; Taheri, M. 5-Fluorouracil: A Narrative Review on the Role of Regulatory Mechanisms in Driving Resistance to This Chemotherapeutic Agent. Front Oncol 2021, 11, 658636, https://doi.org/10.3389/fonc.2021.658636.

27. Oettle, H.; Post, S.; Neuhaus, P.; Gellert, K.; Langrehr, J.; Ridwelski, K.; Schramm, H.; Fahlke, J.; Zuelke, C.; Burkart, C.; Gutberlet, K.; Kettner, E.; Schmalenberg, H.; Weigang-Koehler, K.; Bechstein, W.O.; Niedergethmann, M.; Schmidt-Wolf, I.; Roll, L.; Doerken, B.; Riess, H. Adjuvant chemotherapy with gemcitabine vs observation in patients undergoing curative-intent resection of pancreatic cancer: a randomized controlled trial. JAMA 2007, 297, 267-277, https://doi.org/10.1001/jama.297.3.267.

28. Turpin, A.; el Amrani, M.; Bachet, J-B.; Pietrasz, D.; Schwarz, L.; Hammel, P. Adjuvant Pancreatic Cancer Management: Towards New Perspectives in 2021. Cancers 2020, 12, 3866; https://doi.org/10.3390/cancers12123866.

29. Velu, L.K.P.; Steele, C.W.; Dickson, E.J.; Carter, R.; McKay, C.J.; Horgan, P.G.; McMillan, D.C.; Jamieson, N.B. nab-Paclitaxel plus gemcitabine for metastatic pancreatic cancer: long-term survival from a phase III trial. J Natl Cancer Inst 2015, 107, djv204, https://doi.org/10.1093/jnci/djv204.

30. Harikumar, K.B.; Kunnumakkara, A.B.; Sethi, G., Diagaradjane, P.; Anand, P.; Pandey, M.K.; Gelovani, J.; Krishnan, S.; Guha, S.; Aggarwal, B.B. Resveratrol, a multitargeted agent, can enhance antitumor activity of gemcitabine in vitro and in orthotopic mouse model of human pancreatic cancer. Int J Cancer 2010, 127, 257-268, https://doi.org/10.1002/ijc.25041.

31. Principe, D.; Narbutis, M.; Kumar, S.; Park, A.; Viswakarma,. N.; Dorman, M.J.; Kamath, S.D.; Grippo, P.J.; Fishel, M.L.; Hwang, R.F.; Thummuri, D.; Underwood, P.W.; Munshi, H.G.; Trevino, J.G.; Rana, A. Long-term gemcitabine treatment reshapes the pancreatic tumor microenvironment and sensitizes murine carcinoma to combination immunotherapy. Cancer Res 2020, 80, 3101-3115, https://doi.org/10.1158/0008-5472.CAN-192959.

32. Brooks, M.B. Erlotinib and gefitinib, epidermal growth factor receptor kinase inhibitors, may treat noncancer-related tumor necrosis factor- $\alpha$ mediated inflammatory diseases. Oncologist 2013, 18, e3-e5, https://doi.org/10.1634/theoncologist.2012-0219.

33. Sinn, M.; Bahra, M.; Liersch, T.; Gellert, K.; Messmann, H.; Bechstein, W.; Waldschmidt, D.; Jacobasch, L.; Wilhelm, M.; Rau, B.M.; Grutzmann, R.; Weinmann, A.; Maschmeyer, G.; Pelzer, U.; Stieler, J.M.; Striefler, J.K.; Ghadimi, M.; Bischoff, S.; Dorken, B.; Oettle, H.; Riess, H. CONKO-005: Adjuvant chemotherapy with gemcitabine plus erlotinib versus gemcitabine alone in patients after R0 resection of pancreatic cancer: A multicenter randomized Phase III trial. J Clin Oncol 2017, 35, 3330-3337, https://doi.org/10.1200/JCO.2017.72.6463.

34. Garay, R.P.; Viens, P.; Bauer, J.; Normier, G.; Bardou, M.; Jeannin, J.F.; Chiavaroli, C. Cancer relapse under chemotherapy: why TLR2/4 receptor agonists can help. Eur J Pharmacol 2007, 563, 1-17, https://doi.org/10.1016/j.ejphar.2007.02.018.

35. Laheru, D.; Jaffee, E.M. Immunotherapy for pancreatic cancer - science driving clinical progress. Nat Rev Cancer 2005, 5, 459-467, https://doi.org/10.1038/nrc1630.

36. Akira, S.; Takeda, K. Toll-like receptor signalling. Nat Rev Immunol 2004, 4, 499-511, https://doi.org/10.1038/nri1391. 
37. Takeda, K.; Akira, S. TLR signaling pathways. Semin Immunol 2004, 16, 3-9. https://doi.org/10.1016/j.smim.2003.10.003.

38. Zhao, S.; Zhang, Y.; Zhang, Q.; Wang, F.; Zhang, D. Toll-like receptors and prostate cancer. Front Immunol 2014, 5, 352, https://doi.org/10.3389/fimmu.2014.00352.

39. Durán, N.; Nunes, O.D.S. Characterization of an aggregated polymer from Penicillium sp. (PB-7 Strain). Braz J Med Biol Res 1990, 23, 1289-1302, PMID: 2136563, https://europepmc.org/article/med/2136563.

40. Garcia, P.V.; Seiva, F.R.; Carniato, A.P.; de Mello Junior, W.; Durán, N.; Macedo, A.M.; de Oliveira, A.G.; Romih, R.; Nunes, I.S.; Nunes, O.S.; Favaro, W.J. Increased toll-like receptors and p53 levels regulate apoptosis and angiogenesis in non-muscle invasive bladder cancer: mechanism of action of P-MAPA biological response modifier. BMC Cancer 2016, 16, 422-440, https://doi.org/10.1186/s12885-016-2474-Z.

41. Kim, S.; Toyokawa, H.; Yamao, J.; Satoi, S.; Yanagimoto, H.; Yamamoto, T.; Hirooka, S.; Yamaki, S.; Inoue, K.; Matsui, Y.; Kwon, A.H. Antitumor effect of angiotensin II type 1 receptor blocker losartan for orthotopic rat pancreatic adenocarcinoma. Pancreas https://doi.org/10.1097/MPA.0000000000000125.

42. Hruban, R.H.; Maitra, A.; Goggins, M. Update on pancreatic intraepithelial neoplasia. Int J Clin Exp Pathol 2008, 1, 306-316, https://www.ncbi.nlm.nih.gov/pmc/articles/PMC2480542/.

43. D'Agostini, C.; Pica, F.; Febbraro, G.; Grelli, S.; Chiavaroli, C.; Garaci, E. Antitumour effect of OM-174 and cyclophosphamide on murine B16 melanoma in different experimental conditions. Int Immunopharmacol 2005, 5, 1205-1212, https://doi.org/10.1016/j.intimp.2005.02.013.

44. Ang, L.; Chan, C.P.Y.; Yau, W.P.; Seow, W.J. Association between family history of lung cancer and lung cancer risk: a systematic review and meta-analysis. Lung Cancer. 2020,148, 129-137, https://doi.org/10.1016/j.lungcan.2020.08.012.

45. Dissin, J.; Mills, L.R.; Mains, D.L.; Black, O.Jr.; Webster, P.D. 3rd. Experimental induction of pancreatic adenocarcinoma in rats. J Natl Cancer Inst 1975, 55, 857-864, https://doi.org/10.1093/jnci/55.4.857.

46. Chang, L.S.; Yan, W.L.; Chang, Y.W.; Yeh, Y.C.; Chen, H.W.; Leng, C.H.; Liu, S.J. Gemcitabine enhances antitumor efficacy of recombinant lipoimmunogen-based immunotherapy. Oncoimmunology 2016, 5, 51, https://doi.org/10.1080/2162402X.2015.1095433.

47. Hirooka, Y.; Itoh, A.; Kawashima, H.; Hara, K.; Nonogaki, K.; Kasugai, T.; Ohno, E.; Ishikawa, T.; Matsubara, H.; Ishigami, M.; Katano, Y.; Ohmiya, N.; Niwa, Y.; Yamamoto, K.; Kaneko, T.; Nieda, M.; Yokokawa, K.; Goto, H. A combination therapy of gemcitabine with immunotherapy for patients with inoperable locally advanced pancreatic cancer. Pancreas 2009, 38, 69-74, https://doi.org/10.1097/MPA.0b013e318197a9e3.

48. de Pillis, L.G.; Gu, W.; Radunskaya, A.E. Mixed immunotherapy and chemotherapy of tumors: modeling, applications and biological interpretations. J Theor Biol 2006, 238, 841-862, https://doi.org/10.1016/j.jtbi.2005.06.037.

49. Steinberg, G.D.; Smith, N.D.; Ryder, K.; Strangman, N.M.; Slater, S.J. Factors affecting valrubicin response in patients with bacillus Calmette-Guerin-refractory bladder carcinoma in situ. Postgrad Med 2011, 123, 2834, https://doi.org/10.3810/pgm.2011.05.228.

50. Luo, J.L.; Maeda, S.; Hsu, L.C.; Yagita, H.; Karin, M. Inhibition of NF-kappaB in cancer cells converts inflammation- induced tumor growth mediated by TNFalpha to TRAIL-mediated tumor regression. Cancer Cell 2004, 6, 297-305, https://doi.org/10.1016/j.ccr.2004.08.012.

51. Shankaran, V.; Ikeda, H.; Bruce, A.T.; White, J.M.; Swanson, P.E.; Old, L.J.; Schreiber, R.D. IFNgamma and lymphocytes prevent primary tumour development and shape tumour immunogenicity. Nature 2001, 410, 1107-1111, https://doi.org/10.1038/35074122.

52. Martini, M.; Testi, M.G.; Pasetto, M.; Picchio, M.C.; Innamorati, G.; Mazzocco, M.; Ugel, S.; Cingarlini, S.; Bronte, V.; Zanovello, P.; Krampera, M.; Mosna, F.; Cestari, T.; Riviera, A.P.; Brutti, N.; Barbieri, O.; Matera, L.; Tridente, G.; Colombatti, M.; Sartoris, S. IFN-gamma-mediated upmodulation of MHC class I expression activates tumor-specific immune response in a mouse model of prostate cancer. Vaccine 2010, 28, 3548-3557, https://doi.org/10.1016/j.vaccine.2010.03.007.

53. Alshaker, H.A.; Matalka, K.Z. IFN-gamma, IL-17 and TGF-beta involvement in shaping the tumor microenvironment: The significance of modulating such cytokines in treating malignant solid tumors. Cancer Cell Int 2011, 11, 33, https://doi.org/10.1186/1475-2867-11-33.

54. Tate, D.J. Jr.; Patterson, J.R.; Velasco-Gonzalez, C.; Carroll, E.N.; Trinh, J.; Edwards, D.; Aiyar, A.; FinkelJimenez, B.; Zea, AH. Interferon-gamma-induced nitric oxide inhibits the proliferation of murine renal cell carcinoma cells. Int J Biol Sci 2012, 8, 1109-1120, https://doi.org/10.7150/ijbs.4694.

55. Lamrani, M.; Sassi, N.; Paul, C.; Yousfi, N.; Boucher, J-L.; Gauthier, N.; Labbe, J.; Seignez, C.; Racoeur, C.; Athias, A.; Guerreiro, R.; Vergely, C.; Rochette, L.; Bettaieb, A.; Jeannin, J-F. TLR4/IFN $\gamma$ pathways induce tumor regression via NOS II-dependent NO and ROS production in murine breast cancer models. Oncoimmunology 2015, 5, 26, https://doi.org/10.7150/ijbs.4694. 